\title{
Trace membrane additives affect lipid phases with distinct mechanisms: a modified Ising model
}

\author{
Rebecca L. Meerschaert ${ }^{1}$. Christopher V. Kelly ${ }^{1}$
}

Received: 19 November 2014 / Revised: 25 February 2015 / Accepted: 5 March 2015 / Published online: 28 March 2015

(C) The Author(s) 2015. This article is published with open access at Springerlink.com

\begin{abstract}
The addition of trace molecules into membranes can significantly alter the morphology of the coexisting liquid phases and lipid phase transition temperature. Membrane additives may affect lipid phase dynamics through preferentially partitioning to the boundary between lipid phases or preferentially mixing into one lipid phase. The characteristic differences between these mechanisms are demonstrated here in a minimalistic nearest neighbor model to provide a framework for how slight changes to membrane composition may affect lipid-phase-dependent processes, such as lipid-raft formation, immunological signaling, and molecular sorting preceding endocytosis with coexisting liquid phases. Within the low mole fractions explored here $(\leq 3 \mathrm{~mol} \%)$, increasing the additive concentration linearly changed the phase miscibility temperature. Rotationally asymmetric Janus particles reduced the miscibility transition temperature for all fractions and degree of phase polarization. Rotationally symmetric additives, however, either increased or decreased the phase miscibility temperature depending on the phase preference of the additive. While most experimental molecules may contain aspects of both of these idealized additives, this model provides a broad framework to quantify the effects of membrane additives in regard to lipid phase preference, lipidraft association, and contribution to lipid phase-dependent molecular sorting.
\end{abstract}

Electronic supplementary material The online version of this article (doi:10.1007/s00249-015-1017-x) contains supplementary material, which is available to authorized users.

\section{Christopher V. Kelly}

cvkelly@wayne.edu

1 Department of Physics and Astronomy, Wayne State University, Detroit, MI, USA
Keywords Lipid membrane $\cdot$ Phase transition temperature $\cdot$ Miscibility $\cdot$ Correlation length $\cdot$ Phase partitioning $\cdot$ Simulation

\section{Introduction}

The presence of coexisting liquid-ordered and liquid-disordered lipid phases has been implicated in diverse biological processes with high sensitivity to perturbations in membrane composition, tension, curvature, and temperature (Lingwood et al. 2008). Lipid phase dynamics may inherently provide nanoscale clustering and facilitate lipid-raftmediated processes (Veatch et al. 2008). However, trace membrane additives can disturb the lipid phase dynamics and the associated biological processes. Some additives possess rotational asymmetry, preferentially localize to the phase boundary, reduce the phase miscibility, decrease the miscibility transition temperature $\left(T_{\text {mis }}\right)$, and act as linactants, 1D analogs of surfactants (Trabelsi et al. 2008). Other membrane additives are rotationally symmetric, preferentially localize into one particular lipid phase, and may increase or decrease $T_{\text {mis }}$. Broadly, the effects of membrane additives are quantified by the change to the lipid phase $T_{\text {mis }}$. The difference between a sample temperature and $T_{\text {mis }}$ determines fundamental equilibrium qualities such as the correlation length of the coexisting phases (HonerkampSmith et al. 2008; Palmieri and Safran 2013a) and the dynamics of the phase mobility (Honerkamp-Smith et al. 2012; Palmieri and Safran 2013b).

Numerous molecules have demonstrated potent abilities to alter membrane phase miscibility without necessarily containing a dependence on the rotation of the molecule within the bilayer, including cholesterol (Dietrich et al. 2001; Zhao et al. 2007a; Levental et al. 2009; Heberle 
et al. 2010), fluorophores (Veatch et al. 2007a), anesthetics (Jorgensen et al. 1991; Gray et al. 2013), insecticides (Jorgensen et al. 1991), n-propyl gallate (Zhao et al. 2007b), C-reactive protein (Sáenz et al. 2010), and flavonoids (Ostroumova et al. 2014). Other molecules have been hypothesized to prefer the boundary between two lipid phases because of their molecular structure; for example, hybrid lipids contain one liquid-order-preferring tail and one liquid-disorder-preferring tail (Brewster et al. 2009; Hassan-Zadeh et al. 2014; Li and Gorfe 2014), and asymmetrically lipidated proteins, such as N-Ras and H-Ras, have shown preference for partitioning at the lipid phase boundary (Nicolini et al. 2005; Janosi et al. 2012; Li et al. 2012). Further, molecules such as the nonsteroidal antiinflammatory drug indomethacin may contain both a net phase preference and significant molecular structure asymmetry, although the nanoscale partitioning has yet to be studied (Zhou et al. 2010).

Previous studies have focused on theoretical predictions and experimental examination of systems with a significant fraction of the membrane consisting of phase-polarized particles ( $>10 \mathrm{~mol} \%$ ) (Brewster et al. 2009; Yamamoto et al. 2010; Yamamoto and Safran 2011; Palmieri and Safran 2013a, b; Hassan-Zadeh et al. 2014; Palmieri et al. 2014), whereas this manuscript focuses on small composition changes that can greatly affect membrane phase dynamics and provide a means for live cells to efficiently regulate lipid phase-dependent processes.

The morphology of lipid mixture phase transitions is of the Ising universality class. Monte Carlo simulations of 2D Ising models with conserved order parameters have been used to demonstrate key membrane properties (Frazier et al. 2007; Yethiraj and Weisshaar 2007; HonerkampSmith et al. 2008; Machta et al. 2011, 2012). Within the Ising model, a particle's phase preference $(\sigma)$ is quantified for liquid-disordered (black, $\sigma=-1$ ) or liquid-ordered (white, $\sigma=1$ ) phases, although the particular phase associated with the color and sign of $\sigma$ is arbitrary in this symmetric system. The internal energy between two particles $\left(J_{i, j}\right)$ is equal to $J$ for nearest neighbors and zero otherwise, and it can be calculated from the sum of all particle interactions in the Hamiltonian $(H)$ according to:

$H=-\sum_{\langle i, j\rangle} J_{i, j} \sigma_{i} \sigma_{j}$

When this model contains $50 \%$ white and $50 \%$ black particles on a square lattice, it exhibits critical behavior with $T_{\text {mis }}=2 J /\left(k_{\mathrm{B}} \ln (1+\sqrt{2})\right)$, where $k_{\mathrm{B}}$ is Boltzmann's constant. Isolated plasma membrane vesicles from mast cells happen to be of a composition that demonstrates nearcritical behavior with $T_{\text {mis }}=22{ }^{\circ} \mathrm{C}$ (Veatch et al. 2008), corresponding to a value of $J=0.26 \mathrm{kcal} / \mathrm{mol}$. To relate the complex molecular composition of near-critical membranes to the two-state Ising model, each pixel from the model represents the mean composition of each state. For example, the white particles in the Ising model represent the cell membrane's average liquid-ordered phase composed of lipids with longer, more saturated acyl tails, greater sphingomyelin content, and slightly higher cholesterol content than the black particles. This model permits the observation of complex phase behaviors without explicit incorporation of the molecular details or concentrations of the membrane, as long as the average phase segregation preferences are represented by the value of $J$.

In this manuscript, we demonstrate the influence of trace additives in a two-component nearest neighbor model on a square lattice with conserved order parameters as a means of simulating how additives of differing molecular structure could influence lipid phase mixing. Instead of modifying the value of $J$ to represent changes in membrane composition, a third particle type was incorporated in these simulations to provide greater structural detail of the additive's effect on phase separation and additive partitioning. In a ternary lipid membrane, for example, the additive could be a fourth type of molecule or more of one molecule type that was already present in the membrane. Simulated additives were either rotationally symmetric gray particles or rotational asymmetric phase-polarized particles with gray values of $g$ or polarizations of $p$, respectively (Fig. 1). Generally, the addition of a third type of particle to the system results in a change to the phase diagram, new phase transitions, and variations from critical behavior. Simulations performed with additives of $g=0$ were analogous to the Blume-Emery-Griffiths model (Blume et al. 1971), for which the phase transitions were modeled against an additive concentration with meanfield approximations. However, the additive concentrations explored here are consistent with the composition fluctuations in living and model membranes, including variations in content of cholesterol (Dietrich et al. 2001; Pokorny et al. 2006; Frazier et al. 2007; Zhao et al. 2007a; Levental et al. 2009; Heberle et al. 2010), fluorophores (Veatch et al. 2007a; Frazier et al. 2007), and a variety of other biologically active molecules (Jorgensen et al. 1991; Nicolini et al. 2005; Pokorny et al. 2006; Zhao et al. 2007a, b; Brewster et al. 2009; Sáenz et al. 2010; Zhou et al. 2010; Gray et al. 2013; Ostroumova et al. 2014). While real membranes have been
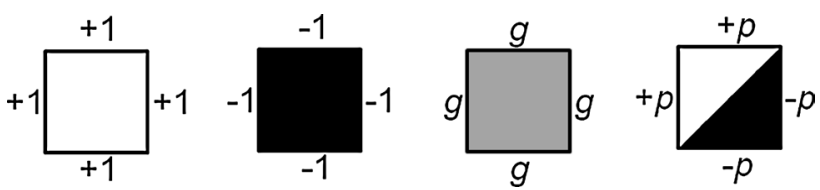

Fig. 1 White, liquid-ordered $(\sigma=1)$; black, liquid-disordered $(\sigma=-1)$; gray particles $(\sigma=g)$ were rotationally symmetric. Phasepolarized additives, or Janus additives, were rotationally asymmetric with a polarized phase preference $(|\sigma|=p)$ 
demonstrated to be near critical in composition (Veatch et al. 2007b, 2008), they have not been shown to be any closer to critical than the additive-included simulations shown here. Further, the experimental method for observing plasma membrane extracts involved the incorporation of fluorescent lipid additives $(<0.25 \mathrm{~mol} \%)$ (Veatch et al. 2008), which altered the membrane composition and presumably its phase behavior but did not diminish the significance or confidence in the result that the membranes were near critical in composition.

This work focuses on small perturbations to the wellunderstood two-state Ising model and demonstrates how trace additives would affect the characteristic size of the co-existing single-phase domains $(\xi)$ and $T_{\text {mis }}$. For $\leq 3$ mol\% additives, $T_{\text {mis }}$ and $\xi$ both changed monotonically with increasing additive concentration, while $T_{\text {mis }}$ changed linearly. Rotationally symmetric particles of $g<1$ and rotationally asymmetric particles of all values of $p$ caused a decrease in both $T_{\text {mis }}$ and $\xi$. However, rotationally symmetric additives of $g>1$ resulted in an increase in both $T_{\text {mis }}$ and $\xi$. The morphological detail provided by this model yields the distributions of additives within the system, which is unavailable from meanfield models. The resulting distributions of the additives at the boundary between phases were quantified, and the applicability of this analysis for assessing experimental membrane additives or cellular perturbations is discussed.

\section{Methods}

Non-local particle exchanges were allowed via a Monte Carlo algorithm conserving the system composition (e.g., $49.5 \%$ white, $49.5 \%$ black, and $1 \%$ additive) and equilibrated for $10^{5}$ global equilibration sweeps on a $512 \times 512$ square grid with biperiodic boundary conditions. An additional $10^{5}$ global sweeps were performed over which an average of the system parameters was reported, e.g., the correlation length. The symmetry of this configuration with equal fractions of white and black particles resulted in the additives having the same effect, regardless of the sign of $g$ or $p$. Further details are provided in the Supplemental Material including a movie of 50 different time points of the equilibrated system.

The correlation length of the phases was calculated from the azimuthal average of the 2D spatial correlation of the system configuration $(I)$ where all white particles were valued at 1 , black particles at 0 , and additives at $(g / 2+0.5)$. Unless otherwise stated, if $p \neq 0$, then $g=0$, and vice versa. The $2 \mathrm{D}$ correlation function $(C)$ as a function of the position $(\bar{r})$ can be calculated by

$C(\bar{r})=\frac{\langle I(\bar{R}) I(\bar{R}+\bar{r})\rangle}{\langle I(\bar{R})\rangle^{2}}$, where \langle\rangle represents the average over all 2D space $(\bar{R})$. Computationally, this was expedited by the use of fast Fourier transforms (FFTs) with

$C(\bar{r})=\frac{\mathrm{FFT}^{-1}\left(|\mathrm{FFT}(I)|^{2}\right)}{N}$,

and normalization $(N)$ (Veatch et al. 2012). The 1D correlation function $(c)$ was computed from

$c(r)=\frac{1}{2 \pi} \int_{0}^{2 \pi} C(r, \theta) \mathrm{d} \theta$,

and the correlation length $(\xi)$ was calculated by nonlinear least square fitting of $c_{\text {fit }}$ to $c(r)$ where

$c_{\text {fit }}=c_{0} \frac{\mathrm{e}^{-r / \xi}}{r^{\eta}}$

and the critical exponent $\eta$ equals 0.25 , which is exact for the Ising model. The critical exponents for the threecomponent systems and finite systems are not equal to that of the Ising model; however, additionally allowing $\eta$ to change did not significantly change fitting results.

Changes to $T_{\text {mis }}$ were measured for various additive fractions and additive types in this minimalistic model. $\Delta T_{\text {mis }}$ was determined by measuring the shift in $\xi$ as a function of temperature and by ascertaining the maximum of the specific heat versus temperature (Veatch et al. 2012) (Figs. S1S3). The finite size effects that alter the perceived transition temperature were consistent for all of these simulations on the same size system, which permitted this simplistic calculation of $\Delta T_{\text {mis }}$ and its broad applicability to systems of other sizes. A demonstration of the independence of these results on system size is presented in the Supplemental Material (Fig. S4).

\section{Results}

Changes in phase miscibility with an increasing additive fraction are visibly obvious in images of the system configuration (Figs. 2, S5, S6). $T_{\text {mis }}$ decreased linearly with increasing fractions of phase-polarized particles, or Janus particles, as predicted from mean-field models (Yethiraj and Weisshaar 2007; Brewster et al. 2009). The miscibility temperature is reduced for any phase polarization $(p)$, while rotationally symmetric gray particles either increased or decreased $T_{\text {mis }}$ depending on their gray value ( $g$ ) (Figs. 3, S6). Increasing $p$ for the phase-polarized additives resulted in a greater decrease in $T_{\text {mis }}$ until $p>3$, upon which the additives segregated into a third phase, separate from the white and black particles, and reduced the white and black particle mixing. Gray particles with $g<1$ encouraged 

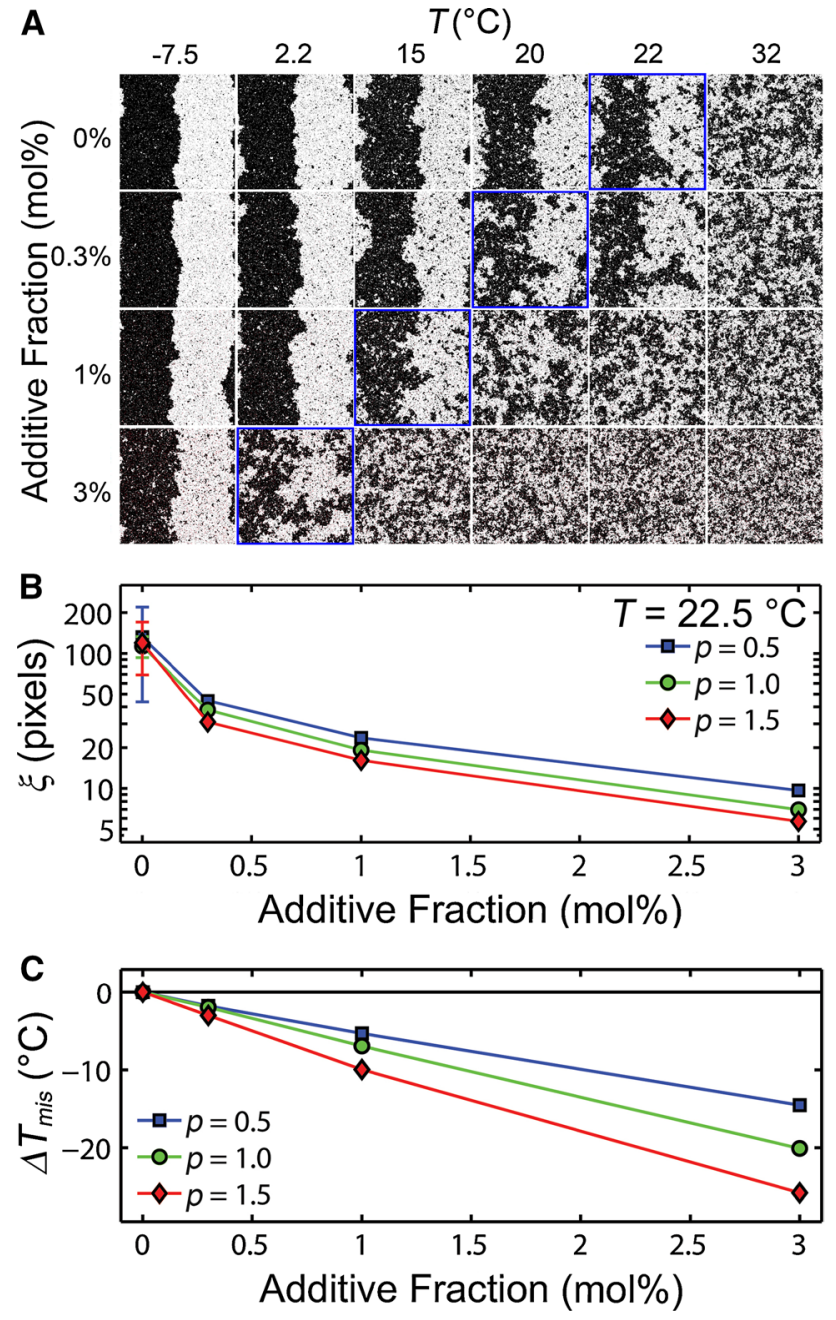

Fig. 2 Increasing the mole fraction of phase-polarized particles caused an increase in the phase miscibility and a decrease of $T_{\text {mis. }}$ a With $p=1$, phase-polarized additives are displayed as red squares in these images, and zoomed-in regions show the individual additives in Fig. S5. Blue outlined images indicate where $T=T_{\text {mis }}$ with $J$ set such that $T_{\text {mis }}$ of the additive-free system $\left(T_{m i s}^{\prime}\right)$ is $22{ }^{\circ} \mathrm{C}$. Increasing the concentration of phase-polarized particles with $p=1$ resulted in a decrease of $\mathbf{b} \xi$ and $\mathbf{c} T_{\text {mis. }} . \Delta T_{\text {mis }}=T_{\text {mis }}-T_{\text {mis }}^{\prime}$. Uncertainty of $T_{m i s}^{\prime}$ was approximately $0.6{ }^{\circ} \mathrm{C}$, and uncertainty of $\xi$ was smaller than the symbol unless otherwise indicated

phase miscibility by decreasing the differences between the ordered and disordered phases, analogous to the incorporation of $n$-alcohols into membranes (Gray et al. 2013). However, when $g>1$, the opposite effect dominates; the difference between the phases was increased when $g>1$ and $T_{\text {mis }}$ was increased, analogous to the addition of $\delta$-lysin to membranes (Pokorny et al. 2006). Gray particles with $g>1$ were no longer of in-between phase and were more liquidorder preferring than white particles, i.e., "super-white."

When $p$ or $g>3$, the additives condensed and formed a distinct phase excluding white or black particles (Fig. S6), and the white-black miscibility temperature became less
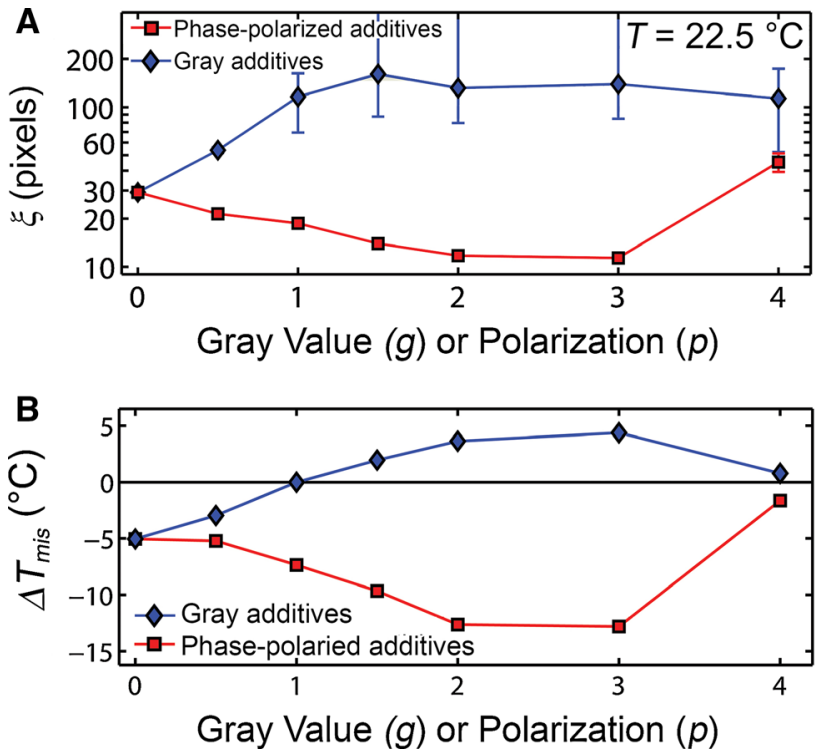

Fig. 3 a $\xi$ and $\mathbf{b} T_{\text {mis }}$ and either increased or decreased upon additive addition depending on the additive properties. $\Delta T_{\text {mis }}=T_{\text {mis }}-T_{m i s}^{\prime}$ where $T_{\text {mis }}^{\prime}=22{ }^{\circ} \mathrm{C}$. Additives comprised $1 \mathrm{~mol} \%$ of the total particles in these simulations. Measurement of $\xi$ is limited by the system size, resulting in large uncertainties for situations with $\xi>100$ pixels (Fig. S4). Images of these systems are included in the Supplemental Material (Figs. S5, S6). $\Delta T_{\text {mis }}$ and $\xi$ changes are symmetrical for negative values of $g$ or $p$

affected by the additives (Fig. 3). For $p$ or $g>3$, simulations were unable to capture a diverse set of configurations for the additive aggregation because of the improbable rearrangement of the condensed phases, analogous to the kinetically trapped configurations that are commonly experienced experimentally. Aggregates of phase-polarized particles at $p=4$ demonstrated rotational ordering of the additives (Fig. S7), which resulted in slower additive diffusion.

The partitioning of additives in each phase and at the domain boundary was measured. A particle was declared to be at the phase boundary if it was adjacent to two white and two black particles. The ratio of additives at the phase boundary to all additives with no adjacent additives was calculated (Fig. 4a). As $p$ increased, the fraction of phasepolarized particles at the phase boundary increased; as $g$ increased, the fraction of gray particles at the phase boundary decreased. Lowering the temperature would have made phase-polarized particles more likely to be found at the phase boundary if the phase morphology had stayed consistent. However, increasing temperature from 8.5 to $36{ }^{\circ} \mathrm{C}$ resulted in $2.3 \pm 0.4$ times more phase boundary (Fig. S8), which was dependent on both simulation size and additive properties, and generally resulted in a higher probability for the additives being located at the phase boundary for higher temperatures. Additive partitioning was normalized to the amount of phase boundary in order to calculate the 
A
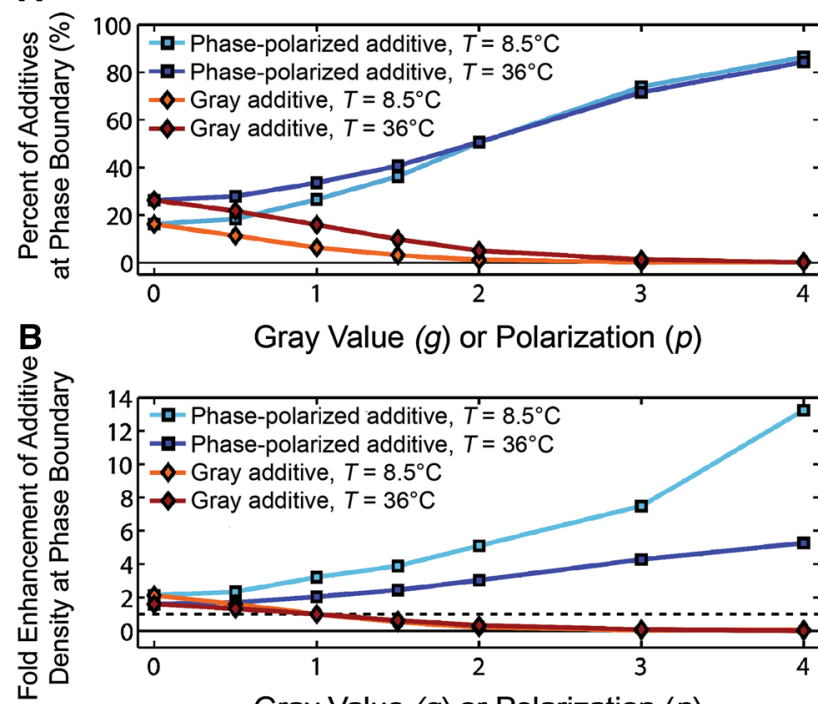

Gray Value $(g)$ or Polarization $(p)$

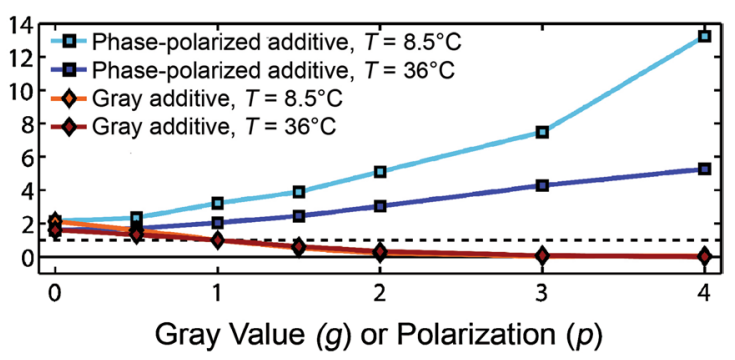

Fig. 4 a The probability that an additive was found at the phase boundary varied with the additive properties and temperature. The fraction of additives at the boundary was calculated as the ratio of the number of additives surrounded by two white and two black particles versus the total number of additives, excluding additives adjacent to other additives. $\mathbf{b}$ The additive locations were normalized by the amount of phase boundary in each condition to yield the fold enhancement of the additive at the phase boundary versus generic white particles. Additives were present at $1 \mathrm{~mol} \%$. Uncertainty was calculated as the standard deviation of different time points and smaller than the symbol unless otherwise indicated

fold enhancement of the additive to the phase boundary relative to the white or black particles (Fig. 4b). Additives more concentrated at the phase boundary than the $g=1$

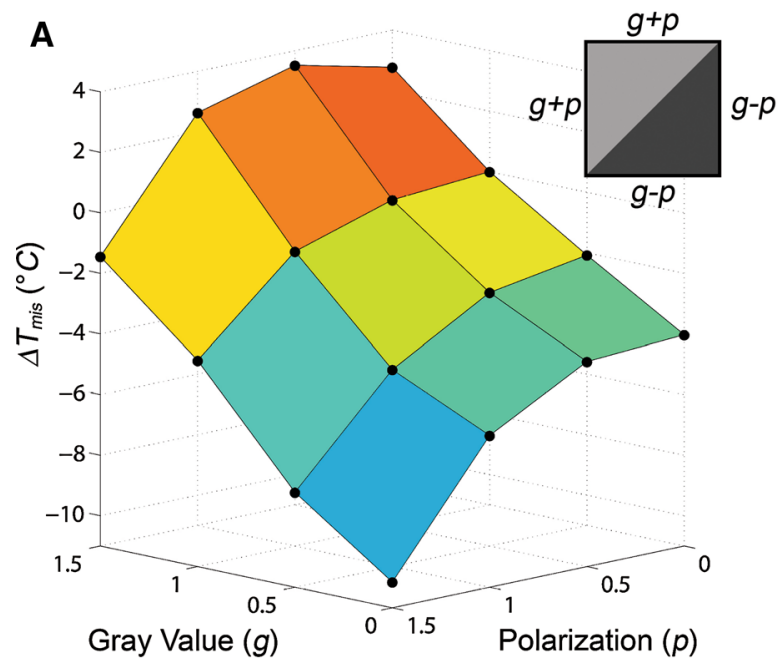

Fig. 5 a Changes to the miscibility transition temperature were induced by the incorporation of $1 \mathrm{~mol} \%$ additives with both net and rotationally asymmetric phase preferences, i.e., dependent on both $g$ particles preferentially partitioned at the phase boundary, whereas additives less concentrated at the phase boundary than the $g=1$ particles preferentially partitioned away from the phase boundary, which is consistent with the sign of $\Delta T_{\text {mis }}$ (Fig. 3). Although there is not a local energy difference for the $g=0$ additive if it is immersed in a single phase or at the phase boundary, there is an energy difference for the system that encourages $g<1$ additives to be concentrated at the phase boundary. At colder temperatures, the phase-polarized additives especially concentrated at the phase boundary because of the decreased entropic drive for mixing.

These results apply to additives in systems that are near critical compositions. It is important to note that these results would vary greatly for compositions that are far from critical compositions because of the increased phase boundary line tension and the increased likelihood for boundary-preferring particles to localize at the boundary in such systems.

In all the prior described simulations, only additives with either $g \neq 0$ or $p \neq 0$ were considered. However, real membrane additives would likely possess both a net phase preference $(g \neq 0)$ and a rotational phase asymmetry $(p \neq 0)$. To predict how changing both $g$ and $p$ for a membrane additive would affect the miscibility transition temperature of the membrane, simulations were performed for additives with rotational asymmetry and a net phase preference at $1 \mathrm{~mol} \%$ (Fig. 5). The changes to the miscibility transition temperature were dependent on both the $g$ and $p$ value of the additive in a cumulative effect. For small values of $g$ and $p$ (i.e., either $g$ or $p \leq 1$ ), $\Delta T_{\text {mis }}$ as a function of $g$ and $p$ was approximately equal to the sum of the

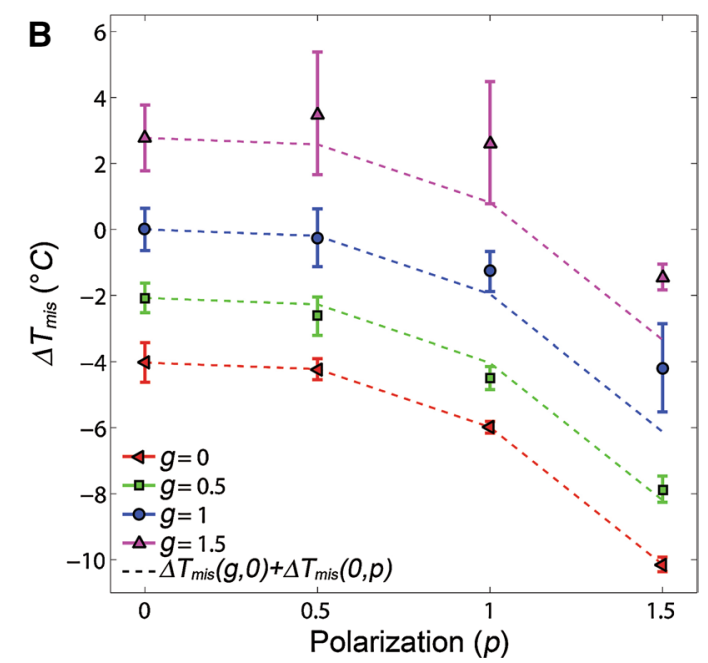

and $p$ values, as shown in the inset. b For small values of $g$ and $p$, $\Delta T_{\text {mis }}$ as a function of $g$ and $p, \Delta T_{\text {mis }}(g, p)$, is approximately equal to $\Delta T_{\text {mis }}(g, 0)+\Delta T_{\text {mis }}(0, p)$ 
change in miscibility temperature from $1 \mathrm{~mol} \%$ gray particles of gray value $g$ plus the change from $1 \mathrm{~mol} \%$ polarized particles of polarization $p$ (Fig. 5b).

\section{Discussion}

Taken together, these idealized membrane additives represent different means by which molecules may alter the lipid phases in a membrane. A membrane-associated molecule may be experimentally quantified with both a $p$ value and $g$ value to describe the effects of the additive molecular structure on lipid phase dynamics in a particular membrane. $g$ is analogous to the partition constant and quantifies the molecule's preference for one phase over the other. $p$ quantifies the molecule's phase asymmetry and preference to partition at the phase boundary. The effects of a particular membrane additive are determined entirely by the membrane's additive-free miscibility temperature ( $T_{m i s}^{\prime}$ ) or internal energy between two particles $(J)$ of the near-critical membrane without a dependence on the particular molecular details of the individual membrane components. The temperatures reported throughout this article could be alternately represented as reduced temperatures $\left(T_{\mathrm{R}}\right)$, where $T_{\mathrm{R}}=\left(T-T_{m i s}^{\prime}\right.$ )/ $T_{\text {mis. }}^{\prime}$. Upon rescaling, these results would be directly applicable to other membranes with different values of $J$ to predict $\Delta T_{\text {mis }}$ for different $T_{\text {mis }}^{\prime}$. Further, experimental use of membranes of varying $T_{m i s}^{\prime}$ would permit the exploration of different $T_{\mathrm{R}}$ values within experimentally limited temperature ranges. For example, increasing the fatty acid tail length in the saturated, liquid-order preferring lipid of a ternary mixture typically increases $T_{m i s}^{\prime}$ and $J$, while decreasing the additive's $g$ value and enhancing the additive's phase boundary preference. Similarly, the $p$ and $g$ values for a membrane additive in a complex cellular membrane could be predicted by measuring the additive's effects on synthetic model membranes of greatly different molecular composition, but similar $T_{m i s}^{\prime}$ and $J$.

Values of $g$ and $p$ that are greater than one represent the phase preferences for the additives that are stronger that then constituents of the phases themselves. For example, if dipalmitoylphosphatidylcholine (DPPC) was added to a dioleoylphosphatidylcholine (DOPC)/dimyristoylphosphatidylcholine (DMPC)/cholesterol bilayer, it would likely have a $g>1$ since DPPC has a stronger preference for the liquid-ordered phase than DOPC, DMPC, or cholesterol. Similarly, it is feasible that a particular phase-preferring component of a hybrid molecule could have a stronger phase preference than average constituents of that phase, i.e., $p>1$. For example, the palmitoyl group of H-Ras or palmitoyloleoylphosphatidylcholine (POPC) could prefer the liquid-ordered phase in a DOPC/DMPC/cholesterol bilayer even more than DOPC, DMPC, or cholesterol.
The quantification of $p$ and $g$ values of cholesterol, for example, requires knowledge of the effects cholesterol addition has on lipid phase separation and the likelihood of cholesterol to be found on the phase boundary. Cholesterol preferentially partitions into liquid-ordered phases, thereby indicating a value of $g>0$. The addition of cholesterol to a membrane typically encourages phase miscibility, indicating that cholesterol has a value of $g<1$. The value of $g$ for cholesterol will vary with the surrounding membrane lipids; however, quantification of cholesterol's effects on the $T_{\text {mis }}$ for near-critical membranes composed of DOPC, DPPC, and cholesterol displayed approximately $15{ }^{\circ} \mathrm{C}$ reduction in $T_{\text {mis }}$ with the addition of $5 \mathrm{~mol} \%$ cholesterol (Veatch et al. 2007b), which implies a $g$ value of 0.3 for cholesterol in this system (i.e., Fig. 3b). The focused quantification of changes in $T_{\text {mis }}$ with variations in cholesterol concentration in near-critical ternary systems would provide greater certainty in the determination of $g$ and $p$.

Similar analysis on the addition of the fluorescent lipid DiI-C12 into membranes composed of DOPC, DPPC, and cholesterol at molar ratios of 35:35:30 demonstrates how a more complex mechanism may also be present (Veatch et al. 2007a). In this system, $T_{\text {mis }}$ increased linearly with increasing DiI-C12 concentrations up to $0.1 \mathrm{~mol} \%$ with reduced effects on $T_{\text {mis }}$ at higher DiI-C12 concentrations. This difference may be due to a particularly drastic effect of DiI-C12 pushing the membrane toward or away from a critical composition (Veatch et al. 2007a) or specific interactions between DiI-C12 and the other lipids in the system.

The theoretical framework presented here provides a basis for the interpretation of experimental data for the lipid phase and boundary preference of membrane-associated molecules. Similarly, this framework provides a means of predicting the lipid-raft association and lipid phase-dependent clustering in complex systems through the extension of results from model membrane studies. This ability to quantify the phase preference of membrane additives may prove to be a valuable parameter for the therapeutic development and evaluation of the therapeutic mechanisms.

Acknowledgments The authors thank Sarah A. Shelby, Paul H. Keyes, and Sarah L. Veatch for conversations and review of the manuscript. This project was funded by laboratory startup funds provided by Wayne State University.

Open Access This article is distributed under the terms of the Creative Commons Attribution License which permits any use, distribution, and reproduction in any medium, provided the original author(s) and the source are credited.

\section{References}

Blume M, Emery VJ, Griffiths RB (1971) Ising model for the $\lambda$ transition and phase separation in He3-He4 mixtures. Phys Rev A 4:1071-1077. doi:10.1103/PhysRevA.4.1071 
Brewster R, Pincus PA, Safran SA (2009) Hybrid lipids as a biological surface-active component. Biophys J 97:1087-1094. doi:10.1016/j.bpj.2009.05.051

Dietrich C, Volovyk ZN, Levi M et al (2001) Partitioning of Thy1, GM1, and cross-linked phospholipid analogs into lipid rafts reconstituted in supported model membrane monolayers. Proc Natl Acad Sci 98:10642-10647. doi:10.1073/pnas.191168698

Frazier ML, Wright JR, Pokorny A, Almeida PFF (2007) Investigation of domain formation in sphingomyelin/cholesterol/POPC mixtures by fluorescence resonance energy transfer and Monte Carlo simulations. Biophys J 92:2422-2433. doi:10.1529/ biophysj.106.100107

Gray E, Karslake J, Machta BB, Veatch SL (2013) Liquid general anesthetics lower critical temperatures in plasma membrane vesicles. Biophys J 105:2751-2759. doi:10.1016/j.bpj.2013.11.005

Hassan-Zadeh E, Baykal-Caglar E, Alwarawrah M, Huang J (2014) Complex roles of hybrid lipids in the composition, order, and size of lipid membrane domains. Langmuir 30:1361-1369. doi:10.1021/la4044733

Heberle FA, Wu J, Goh SL et al (2010) Comparison of three ternary lipid bilayer mixtures: FRET and ESR reveal nanodomains. Biophys J 99:3309-3318. doi:10.1016/j.bpj.2010.09.064

Honerkamp-Smith AR, Cicuta P, Collins MD et al (2008) Line tensions, correlation lengths, and critical exponents in lipid membranes near critical points. Biophys J 95:236-246. doi:10.1529/ biophysj.107.128421

Honerkamp-Smith AR, Machta BB, Keller SL (2012) Experimental observations of dynamic critical phenomena in a lipid membrane. Phys Rev Lett 108:265702

Janosi L, Li Z, Hancock JF, Gorfe AA (2012) Organization, dynamics, and segregation of Ras nanoclusters in membrane domains. Proc Natl Acad Sci. doi:10.1073/pnas.1200773109

Jorgensen K, Ipsen J, Mouritsen O et al (1991) The Effects of density-fluctuations on the partitioning of foreign molecules into lipid bilayers-application to anesthetics and insecticides. Biochim Biophys Acta 1067:241-253. doi:10.1016/0005-2736(91)90050-I

Levental I, Byfield FJ, Chowdhury P et al (2009) Cholesterol-dependent phase separation in cell-derived giant plasma-membrane vesicles. Biochem J 424:163-167. doi:10.1042/BJ20091283

Li Z, Gorfe AA (2014) Modulation of a small two-domain lipid vesicle by linactants. J Phys Chem B. doi:10.1021/jp5042525

Li Z, Janosi L, Gorfe AA (2012) Formation and domain partitioning of H-ras peptide nanoclusters: effects of peptide concentration and lipid composition. J Am Chem Soc 134:17278-17285. doi:10.1021/ja307716z

Lingwood D, Ries J, Schwille P, Simons K (2008) Plasma membranes are poised for activation of raft phase coalescence at physiological temperature. Proc Natl Acad Sci 105:10005-10010. doi:10.1073/pnas.0804374105

Machta BB, Papanikolaou S, Sethna JP, Veatch SL (2011) Minimal model of plasma membrane heterogeneity requires coupling cortical actin to criticality. Biophys J 100:1668-1677. doi:10.1016/j. bpj.2011.02.029

Machta BB, Veatch SL, Sethna JP (2012) Critical Casimir forces in cellular membranes. Phys Rev Lett 109:138101. doi:10.1103/ PhysRevLett.109.138101

Nicolini C, Baranski J, Schlummer S et al (2005) Visualizing association of N-Ras in lipid microdomains: influence of domain structure and interfacial adsorption. J Am Chem Soc 128:192-201. doi:10.1021/ja055779x
Ostroumova OS, Chulkov EG, Stepanenko OV, Schagina LV (2014) Effect of flavonoids on the phase separation in giant unilamellar vesicles formed from binary lipid mixtures. Chem Phys Lipids 178:77-83. doi:10.1016/j.chemphyslip.2013.12.005

Palmieri B, Safran SA (2013a) Hybrid lipids increase the probability of fluctuating nanodomains in mixed membranes. Langmuir 29:5246-5261. doi:10.1021/la4006168

Palmieri B, Safran SA (2013b) Hybrid lipids increase nanoscale fluctuation lifetimes in mixed membranes. Phys Rev E 88:032708. doi:10.1103/PhysRevE.88.032708

Palmieri B, Grant M, Safran SA (2014) Prediction of the dependence of the line tension on the composition of linactants and the temperature in phase separated membranes. Langmuir 30:1173411745. doi:10.1021/la502347a

Pokorny A, Yandek LE, Elegbede AI et al (2006) Temperature and composition dependence of the interaction of $\delta$-lysin with ternary mixtures of sphingomyelin/cholesterol/POPC. Biophys J 91:2184-2197. doi:10.1529/biophysj.106.085027

Sáenz A, López-Sánchez A, Mojica-Lázaro J et al (2010) Fluidizing effects of C-reactive protein on lung surfactant membranes: protective role of surfactant protein A. FASEB J Off Publ Fed Am Soc Exp Biol 24:3662-3673. doi:10.1096/fj.09-142646

Trabelsi S, Zhang S, Lee TR, Schwartz DK (2008) Linactants: surfactant analogues in two dimensions. Phys Rev Lett 100:037802. doi:10.1103/PhysRevLett.100.037802

Veatch SL, Leung SSW, Hancock REW, Thewalt JL (2007a) Fluorescent probes alter miscibility phase boundaries in ternary vesicles. J Phys Chem B 111:502-504. doi:10.1021/jp067636i

Veatch SL, Soubias O, Keller SL, Gawrisch K (2007b) Critical fluctuations in domain-forming lipid mixtures. Proc Natl Acad Sci USA 104:17650-17655. doi:10.1073/pnas.0703513104

Veatch SL, Cicuta P, Sengupta P et al (2008) Critical fluctuations in plasma membrane vesicles. ACS Chem Biol 3:287-293. doi:10.1021/cb800012x

Veatch SL, Machta BB, Shelby SA et al (2012) Correlation functions quantify super-resolution images and estimate apparent clustering due to over-counting. PLoS one 7:e31457. doi:10.1371/journal.pone. 0031457

Yamamoto T, Safran SA (2011) Line tension between domains in multicomponent membranes is sensitive to degree of unsaturation of hybrid lipids. Soft Matter 7:7021. doi:10.1039/c0sm00882f

Yamamoto T, Brewster R, Safran SA (2010) Chain ordering of hybrid lipids can stabilize domains in saturated/hybrid/cholesterol lipid membranes. EPL Europhys Lett 91:28002. doi:10.1209/0295-5075/91/28002

Yethiraj A, Weisshaar JC (2007) Why are lipid rafts not observed in vivo? Biophys J 93:3113-3119. doi:10.1529/ biophysj.106.101931

Zhao J, Wu J, Heberle FA et al (2007a) Phase studies of model biomembranes: complex behavior of DSPC/DOPC/cholesterol. Biochim Biophys Acta 1768:2764-2776. doi:10.1016/j. bbamem.2007.07.008

Zhao J, Wu J, Shao H et al (2007b) Phase studies of model biomembranes: macroscopic coexistence of $\mathrm{L} \alpha+\mathrm{L} \beta$, with lightinduced coexistence of $\mathrm{L} \alpha+$ Lo phases. Biochim Biophys Acta 1768:2777-2786. doi:10.1016/j.bbamem.2007.07.009

Zhou Y, Plowman SJ, Lichtenberger LM, Hancock JF (2010) The anti-inflammatory drug indomethacin alters nanoclustering in synthetic and cell plasma membranes. J Biol Chem 285:35188 35195. doi:10.1074/jbc.M110.141200 